\title{
Planar photonic crystal resonant cavities
}

\section{Kartik Srinivasan, Oskar J. Painter}

Kartik Srinivasan, Oskar J. Painter, "Planar photonic crystal resonant cavities," Proc. SPIE 4870, Active and Passive Optical Components for WDM Communications II, (11 July 2002); doi: 10.1117/12.475544

Event: ITCom 2002: The Convergence of Information Technologies and SPIE. Communications, 2002, Boston, MA, United States 


\title{
Planar Photonic Crystal Resonant Cavities
}

\author{
Kartik Srinivasan and Oskar Painter \\ Department of Applied Physics, California Institute of Technology, Pasadena, CA 91125, USA.
}

\begin{abstract}
Optically thin dielectric slabs, in which a fully etched through two-dimensional patterning is applied, can be used to form high-Q optical cavities with modal volumes approaching the theoretical limit of a cubic half-wavelength. A cavity design strategy based upon simple group theoretical techniques is presented in which emphasis is placed upon a momentum space description of the resonant modes. It is shown that photonic crystal laser cavities can be designed with a particular wavelength, polarization, and radiation pattern using these methods.
\end{abstract}

Keywords: photonic crystal, microcavity, semiconductor laser, InGaAsP

\section{INTRODUCTION}

Planar photonic crystal (PC) waveguide structures ${ }^{1-3}$ have been the subject of intense research activity in recent years, as they hold a great deal of promise for use in future generations of high-density lightwave circuits. Significant progress has been made in the study of in-plane waveguides ${ }^{4-7}$ coupled-resonator-waveguide systems,${ }^{8-10}$ and add-drop devices. ${ }^{11,12}$ One particular area of interest is the study of optical nanocavities in two-dimensional (2D) PC slab waveguides, where the strong dispersion of the photonic lattice can be used to trap light in volumes close to the theoretical limit of a cubic half-wavelength. ${ }^{13-16}$ Mature fabrication technologies such as planar lithography provide precise control of their salient properties, including size, wavelength, emission direction, and polarization of resonant modes. As a result, these structures are well-suited for applications beyond lightwave technologies, such as for cavity-QED studies of coherent electron-photon interactions. Thus, the development of basic design methods for PC nanocavities is of considerable importance. In particular, by considering the photonic crystal modes in Fourier Space, basic group theoretical techniques can be applied to elucidate and predict fundamental characteristics of the defect cavities. These techniques are used as a precursor to more intensive numerical calculations, such as those performed through the finite-difference time-domain (FDTD) method. The qualitative understanding given by the simple symmetry analysis serves as a powerful guide when undertaking more detailed investigations of the resonant mode properties of these nanocavities.

In this paper, we examine the resonant mode properties of intentionally introduced localized defects within 2D PC slab waveguides. The cavity of interest here is composed of an optically thin dielectric slab surrounded by air and patterned with a 2D hexagonal array of holes (Figure 1(a)). A defect is created by altering the dielectric constant in a small region of space in hopes of trapping light within the defect region. This can be achieved in practice by varying the radius of the central hole, or by removing a hole entirely and varying the nearest neighbor holes. By changing the nature of the defect in such a manner, one can profoundly affect the properties of the resonant cavity modes. In particular, the polarization and radiation pattern of the emitted modes can be largely controlled by design of a suitable defect cavity.

The design and analysis of a given defect cavity begins with a study of the symmetries present in the system. Simple group theoretical techniques are used to produce approximate forms for the resonant modes of the cavity. In addition, the symmetry analysis provides information on the transformation properties of these resonant modes, as well as a scheme for labeling them. The results of the group theory analysis are extended through numerical calculations using the FDTD method. ${ }^{14}$ FDTD allows for a detailed investigation of the emission characteristics of the PC cavities, including their spectral response, resonant mode field patterns, and loss properties. ${ }^{17}$

Further author information: (Send correspondence to K.S.) K.S.: E-mail: kartik@caltech.edu, Telephone: 16263956269 


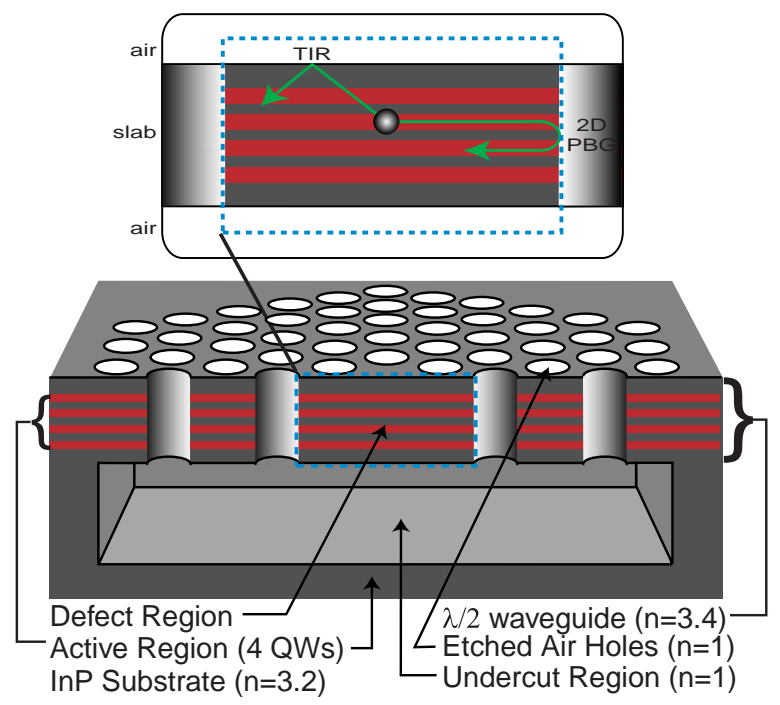

(a) PC slab waveguide.

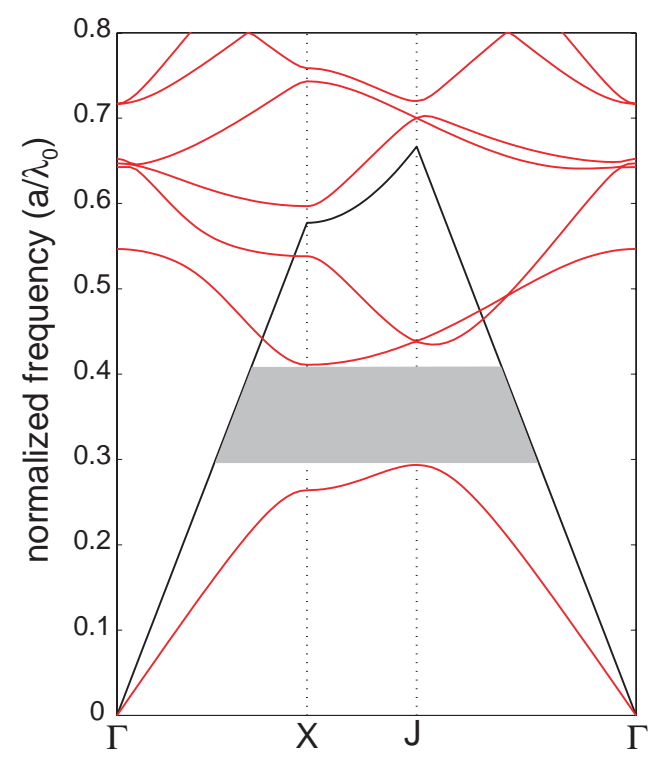

(b) In-plane bandstructure.

Figure 1. (a) Illustration of the two-dimensional hexagonal PC slab waveguide structure. (b) Fundamental TE-like (even) guided mode bandstructure $\left(r / a=0.36, n_{\text {slab }}=n_{\text {eff }}=2.65\right)$. The guided mode band-gap extends over a normalized frequency of $0.29-0.41$. The air (cladding) light line is shown as a solid black line.

The following sections detail a study of the optical properties of experimentally realized defect cavities of varying symmetry in a $2 \mathrm{D} \mathrm{PC}$ slab waveguide. Section 2 outlines the application of simple group theory in producing approximate results describing many of the salient properties of the PC defect cavities. Section 3 presents experimental data in the form of spectroscopic probing through photoluminescence (PL) measurements of fabricated devices. In addition, some results of FDTD simulations are given, establishing a clear correspondence with the group theory. Polarization measurements of the emitted PL further support the predictions made by the group theory and numerical analyses.

\section{SYMMETRY ANALYSIS}

The spatial symmetries within Maxwell's equations are determined by the translation and rotary-reflection symmetries of the dielectric function, $\epsilon(\mathbf{r}) .^{18,19}$ As such, group theory can be used to predict and categorize the resonant modes of defects within PC structures. A two step process is implemented here. The unperturbed slab waveguide modes are first used to generate approximate field patterns for the PC modes at the band-edges defining the guided mode band-gap. The PC band-edge states are then used as a symmetry basis to generate approximate forms for the localized defect modes lying within the band-gap.

\subsection{Band-edge modes}

The modes of a symmetric slab waveguide, patterned or unpatterned, separate into modes of even or odd parity with respect to a mirror plane in the middle of the dielectric slab. Of interest here are the fundamental guided modes, which for the slab thicknesses of the devices analyzed in section 3 , have a wavelength commensurate with the emission band of the quantum-wells. This effectively reduces the spatial dimension of the problem from three to two. In the mirror plane of the waveguide, the fundamental even and odd modes can be represented by scalar fields $B_{z}$ and $E_{z}$, respectively. 


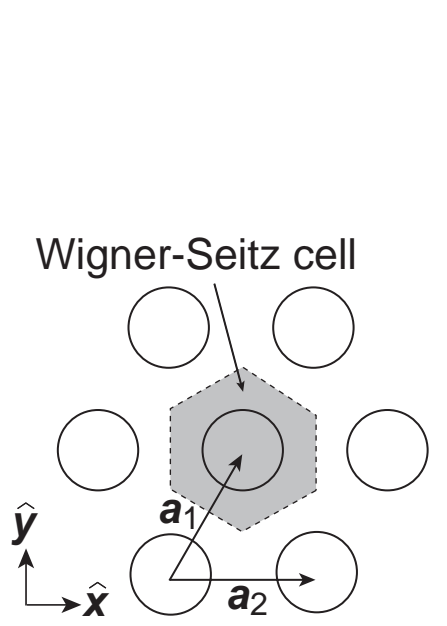

(a) Real space lattice. $\left|\mathbf{a}_{1}\right|=\left|\mathbf{a}_{2}\right|=a$.

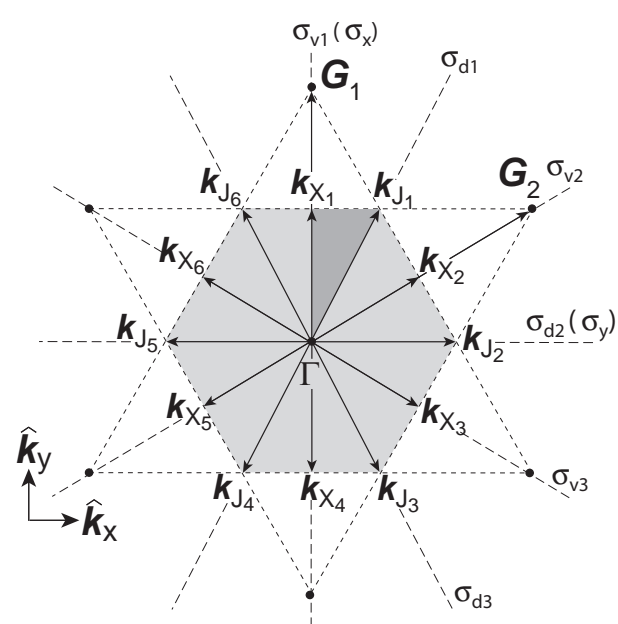

(b) Reciprocal space. $\left|\mathbf{G}_{1}\right|=\left|\mathbf{G}_{2}\right|=$ $4 \pi / \sqrt{3} a,\left|\mathbf{k}_{X}\right|=2 \pi / \sqrt{3} a,\left|\mathbf{k}_{J}\right|=$ $4 \pi / 3 a$.

Figure 2: Illustration of the real and reciprocal spaces of the two-dimensional hexagonal PC.

For the hexagonal photonic lattice of the cavities studied here a band-gap opens up in the frequency spectrum of the fundamental even guided modes, but not in the fundamental odd mode spectrum. ${ }^{14}$ For this reason we focus on the fundamental even modes (TE-like) of the slab waveguide, whose magnetic field patterns within the mirror plane of an unpatterned slab are given by $\mathbf{B}_{\mathbf{k}_{\perp}}\left(\mathbf{r}_{\perp}\right)=\hat{z} e^{-i\left(\mathbf{k}_{\perp} \cdot \mathbf{r}_{\perp}\right)}$ for in-plane momentum $\mathbf{k}_{\perp}$ and coordinates within the mirror plane $\mathbf{r}_{\perp}$. For a $2 \mathrm{D}$ photonic crystal (with or without vertical waveguiding), the point group symmetry of the hexagonal photonic crystal is $D_{6 h}$. Narrowing our scope to TE-like modes of a symmetric slab, the point group symmetry of the system can be effectively reduced to $C_{6 v}=D_{6 h} / \sigma_{h}$, where the horizontal mirror plane $\left(\sigma_{h}\right)$ lies in the middle of the dielectric slab. A plot of the approximate* in-plane bandstructure for the fundamental TE-like guided modes of a half-wavelength thick hexagonally patterned slab waveguide is given in Figure 1(b).

Upon patterning the slab waveguide, coupling occurs between waveguide modes with similar unperturbed frequencies and similar propagation constants modulo $\mathbf{G}$, where $\mathbf{G}$ is a reciprocal lattice vector. The high symmetry points of the first Brillouin zone (IBZ) are the six $X$-points $\left(\left\{ \pm(0,1) k_{X}, \pm(\sqrt{3} / 2,1 / 2) k_{X}, \pm(\sqrt{3} / 2,-1 / 2) k_{X}\right\}\right)$, the six $J$-points $\left(\left\{ \pm(1 / 2, \sqrt{3} / 2) k_{J}, \pm(1 / 2,-\sqrt{3} / 2) k_{J}, \pm(1,0) k_{J}\right\}\right)$, and the $\Gamma$-point $=(0,0)$. The first-order bandgap of the hexagonal lattice (see Figure $1(\mathrm{~b})$ ) is defined from above by the $X$-point ("conduction" band-edge) and below by the $J$-point ("valence" band-edge). The different degenerate $X$ and degenerate $J$ points in the IBZ are labelled as in Figure 2(b). The group of the wave vector at the $X, J$, and $\Gamma$ points is $C_{2 v}, C_{3 v}$, and $C_{6 v}$, respectively. Character tables ${ }^{20}$ for these groups are given in Table 1.

\subsubsection{X-point}

From Bloch's theorem we know the eigenmodes at the $X$-point of a hexagonal photonic crystal must be the product of a periodic function and a plane wave with momentum $\mathbf{k}_{X}$. In the photonic crystal structures studied here, we are interested in wavelengths near the first order band-gap. These wavelengths correspond to the lowest frequency modes at the $X$-point, which in the unperturbed waveguide have a frequency $\omega_{o}^{X} \approx c\left|\mathbf{k}_{X}\right| / n_{\text {eff }}$, where

${ }^{*}$ In this calculation a $2 \mathrm{D}$ hexagonal PC with host dielectric constant given by the effective index of the fundamental TE mode of the half-wavelength thick slab is analyzed. ${ }^{14}$ 
Table 1: Point Group character tables.

\begin{tabular}{c|rrrrrr}
\hline$C_{6 v}$ & $E$ & $C_{2}$ & $2 C_{3}$ & $2 C_{6}$ & $3 \sigma_{d}$ & $3 \sigma_{v}$ \\
\hline$A_{1}^{\prime \prime}$ & 1 & 1 & 1 & 1 & 1 & 1 \\
$A_{2}^{\prime \prime}$ & 1 & 1 & 1 & 1 & -1 & -1 \\
$B_{1}^{\prime \prime}$ & 1 & -1 & 1 & -1 & 1 & -1 \\
$B_{2}^{\prime \prime}$ & 1 & -1 & 1 & -1 & -1 & 1 \\
$E_{1}$ & 2 & -2 & -1 & 1 & 0 & 0 \\
$E_{2}$ & 2 & 2 & -1 & -1 & 0 & 0 \\
\hline$S_{d 1}$ & 3 & -3 & 0 & 0 & 1 & -1 \\
$S_{a 1}$ & 2 & 0 & 2 & 0 & -2 & 0 \\
$S_{a 2}$ & 3 & 3 & 0 & 0 & -1 & -1
\end{tabular}

\begin{tabular}{c|rrrr}
\hline$C_{2 v}$ & $E$ & $C_{2}$ & $\sigma_{x}\left(\sigma_{v 1}\right)$ & $\sigma_{y}\left(\sigma_{d 2}\right)$ \\
\hline$A_{1}$ & 1 & 1 & 1 & 1 \\
$A_{2}$ & 1 & 1 & -1 & -1 \\
$B_{1}$ & 1 & -1 & -1 & 1 \\
$B_{2}$ & 1 & -1 & 1 & -1 \\
\hline$S_{d 1}$ & 3 & -3 & -1 & 1 \\
$S_{a 1}$ & 2 & 0 & 0 & -2 \\
$S_{a 2}$ & 3 & 3 & -1 & -1 \\
\multicolumn{5}{|c}{} \\
\hline$C_{3 v}$ & $E$ & $2 C_{3}$ & $3 \sigma_{d}$ & \\
\hline$A_{1}^{\prime}$ & 1 & 1 & 1 & \\
$A_{2}^{\prime}$ & 1 & 1 & -1 & \\
$E$ & 2 & -1 & 0 &
\end{tabular}

the guided mode has an effective index $n_{e f f}$ and a scalar field within the symmetry plane of the slab waveguide given by $\mathbf{B}=\hat{z} e^{-i \mathbf{k}_{X_{1}} \cdot \mathbf{r}_{\perp}}$.

A symmetry basis for the modes of the patterned slab waveguide at the $X$-point (irreducible representations (IRREPs) of the little group at the $X$-point) can be found by applying the symmetry operations of the group of the wave vector $\left(\mathcal{G}_{o \mathbf{k}_{X}}=C_{2 v}\right)$ to the seed vector $\mathbf{B}_{\mathbf{k}_{X_{1}}}$. In this case, the basis is simply $\left(\mathbf{B}_{\mathbf{k}_{X_{1}}}, \mathbf{B}_{-\mathbf{k}_{X_{1}}}\right)$. Projecting this symmetry basis onto the IRREP spaces of $C_{2 v}$ yields:

$$
\begin{aligned}
& \mathbf{B}_{A_{2}}^{X_{1}}=\hat{z} \cos \left(\mathbf{k}_{X_{1}} \cdot \mathbf{r}_{\perp}\right) \\
& \mathbf{B}_{B_{1}}^{X_{1}}=\hat{z} \sin \left(\mathbf{k}_{X_{1}} \cdot \mathbf{r}_{\perp}\right)
\end{aligned}
$$

where $A_{2}$ and $B_{1}$ label the IRREP spaces of $C_{2 v}$ (see Table 1). Considering that the dielectric structure, as defined here, has a low index air hole at the origin, then $\mathbf{B}_{A_{2}}^{X_{1}}$ corresponds to the "valence" band mode and $\mathbf{B}_{B_{1}}^{X_{1}}$ to the "conduction" band mode. ${ }^{21}$

In order to fully define the modes at the $X$-point all modes of the star of $\mathbf{k}(\star \mathbf{k})$ must be included. The result is the following set of degenerate valence band modes,

$$
V B_{A_{2}}^{X}=\hat{z}\left(\begin{array}{c}
\cos \left(\mathbf{k}_{X_{1}} \cdot \mathbf{r}_{\perp}\right) \\
\cos \left(\mathbf{k}_{X_{2}} \cdot \mathbf{r}_{\perp}\right) \\
\cos \left(\mathbf{k}_{X_{3}} \cdot \mathbf{r}_{\perp}\right)
\end{array}\right)
$$

and degenerate conduction band modes,

$$
C B_{B_{1}}^{X}=\hat{z}\left(\begin{array}{c}
\sin \left(\mathbf{k}_{X_{1}} \cdot \mathbf{r}_{\perp}\right) \\
\sin \left(\mathbf{k}_{X_{2}} \cdot \mathbf{r}_{\perp}\right) \\
\sin \left(\mathbf{k}_{X_{3}} \cdot \mathbf{r}_{\perp}\right)
\end{array}\right)
$$




\subsubsection{J-point}

A similar procedure may be performed in order to determine approximate forms for the TE-like valence and conduction band modes at the $J$-point of the IBZ. An approximate form for the valence band-edge and conduction band-edge modes at the $J$-point are:

$$
\begin{gathered}
V B_{A_{2}^{\prime}}^{J}=\hat{z}\left(\begin{array}{c}
e^{-i \mathbf{k}_{J_{1}} \cdot \mathbf{r}_{\perp}}+e^{-i \mathbf{k}_{J_{3}} \cdot \mathbf{r}_{\perp}}+e^{-i \mathbf{k}_{J_{5}} \cdot \mathbf{r}_{\perp}} \\
e^{-i \mathbf{k}_{J_{2}} \cdot \mathbf{r}_{\perp}}+e^{-i \mathbf{k}_{J_{4}} \cdot \mathbf{r}_{\perp}}+e^{-i \mathbf{k}_{J_{6}} \cdot \mathbf{r}_{\perp}}
\end{array}\right), \\
C B_{E}^{J}=\hat{z}\left(\begin{array}{c}
e^{-i \mathbf{k}_{J_{1}} \cdot \mathbf{r}_{\perp}}+e^{-i \mathbf{k}_{J_{3}} \cdot \mathbf{r}_{\perp}}-2 e^{-i \mathbf{k}_{J_{5}} \cdot \mathbf{r}_{\perp}} \\
e^{-i \mathbf{k}_{J_{1}} \cdot \mathbf{r}_{\perp}}-e^{-i \mathbf{k}_{J_{3}} \cdot \mathbf{r}_{\perp}} \\
e^{-i \mathbf{k}_{J_{2}} \cdot \mathbf{r}_{\perp}}+e^{-i \mathbf{k}_{J_{4}} \cdot \mathbf{r}_{\perp}}-2 e^{-i \mathbf{k}_{J_{6}} \cdot \mathbf{r}_{\perp}} \\
e^{-i \mathbf{k}_{J_{2}} \cdot \mathbf{r}_{\perp}}-e^{-i \mathbf{k}_{J_{4}} \cdot \mathbf{r}_{\perp}}
\end{array}\right),
\end{gathered}
$$

where $A_{2}^{\prime}$ and $E$ are IRREP labels describing how each symmetry basis transforms under $C_{3 v}$, the point group at the $J$-point.

\subsection{Conduction Band Donor Modes}

By perturbing the dielectric constant in a small region of a periodic photonic crystal lattice, one mixes the Bloch modes of the lattice and localized defect modes can form. If the perturbation corresponds to a local increase in the dielectric constant, then the localized modes are formed predominantly from the conduction band modes, specifically the modes at the band-edge. This is a result of the fact that mode frequencies decrease with an increase in the dielectric constant. ${ }^{21}$ Thus, the conduction band-edge modes are "pulled" into the band-gap of the photonic crystal near the defect, resulting in a localized state. This type of localized mode will be termed a donor mode in analogy to the electronic defect states in crystalline materials.

For the hexagonal PC lattice the minimum in the conduction band occurs at the $X$-point (see Figure 1(b)). Therefore, the appropriate symmetry basis to use for describing localized donor modes is the degenerate conduction band-edge modes of $C B_{B_{1}}^{X}$. The simplest defect is that of a single missing air hole in the photonic lattice. By removing a hole we have destroyed the translational symmetry of the photonic lattice and are left with its point group symmetry, $C_{6 v}$.

The matrix representation of the $C B_{B_{1}}^{X}$ basis under the operations of $C_{6 v}$ is labelled $S_{d 1}$, and its characters are shown in Table 1. From the $C_{6 v}$ character table we find that $S_{d 1}=E_{1} \oplus B_{1}^{\prime \prime}$. Using the projection operators on $C B_{B_{1}}^{X}$, a set of basis functions for $E_{1}$ and $B_{1}^{\prime \prime}$ looks as follows:

$$
\begin{aligned}
\mathbf{B}_{B_{1}^{\prime \prime}}^{d 1} & =\hat{z}\left(\sin \left(\mathbf{k}_{X_{1}} \cdot \mathbf{r}_{\perp}\right)-\sin \left(\mathbf{k}_{X_{2}} \cdot \mathbf{r}_{\perp}\right)+\sin \left(\mathbf{k}_{X_{3}} \cdot \mathbf{r}_{\perp}\right)\right), \\
\mathbf{B}_{E_{1}, 1}^{d 1} & =\hat{z}\left(2 \sin \left(\mathbf{k}_{X_{1}} \cdot \mathbf{r}_{\perp}\right)+\sin \left(\mathbf{k}_{X_{2}} \cdot \mathbf{r}_{\perp}\right)-\sin \left(\mathbf{k}_{X_{3}} \cdot \mathbf{r}_{\perp}\right)\right), \\
\mathbf{B}_{E_{1}, 2}^{d 1} & =\hat{z}\left(\sin \left(\mathbf{k}_{X_{2}} \cdot \mathbf{r}_{\perp}\right)+\sin \left(\mathbf{k}_{X_{3}} \cdot \mathbf{r}_{\perp}\right)\right) .
\end{aligned}
$$

The $\left(d 1, B_{1}^{\prime \prime}\right)$ donor mode transforms like a hexapole, whereas the degenerate $\left(d 1, E_{1}\right)$ modes transform as an $(x, y)$-dipole pair.

By introducing defect regions with lower symmetry than the host photonic lattice one is able to remove degeneracies in the localized mode spectrum. The $X$-split and $Y$-split cavities studied below have a defect region with $C_{2 v}$ symmetry as opposed to the full $C_{6 v}$ symmetry of the lattice. The effects of this symmetry lowering can be quite easily determined using group theory by looking at the compatibility relations between the IRREPs of the full and reduced symmetry groups: 


$$
\begin{aligned}
C_{6 v} & \rightarrow C_{2 v} \\
\mathbf{B}_{B_{1}^{\prime \prime}}^{d 1} & \rightarrow \mathbf{B}_{B_{1}}^{d 1,1}, \\
\mathbf{B}_{E_{1}, 1}^{d 1} & \rightarrow \mathbf{B}_{B_{1}}^{d 1,2}(x \text {-dipole }), \\
\mathbf{B}_{E_{1}, 2}^{d 1} & \rightarrow \mathbf{B}_{B_{2}}^{d 1}(y \text {-dipole }) .
\end{aligned}
$$

In the case of the $X$ and $Y$-split cavities with $C_{2 v}$ symmetry, group theory predicts the splitting of the degenerate dipole-like modes into $x$ and $y$ dipole-like modes with differing frequencies. This is born out both in the numerical simulations and in the experimental measurements of section 3 below.

\subsection{Valence Band Acceptor Modes}

If the dielectric constant had been reduced in a small region within the photonic lattice, by enlarging an air hole for instance, then instead of pulling the conduction band modes down into the photonic crystal band-gap the valence band modes are "pushed" up into the band-gap. In this case modes localized to the defect region are formed predominantly from mixtures of Bloch modes from the valence band-edge. This type of defect mode is termed an acceptor mode, again in analogy to the electronic states in a crystal. For the hexagonal lattice the maximum of the valence band occurs at the $J$-point (see Figure 1(b)). As in the previous section, the obvious symmetry basis to use to describe the acceptor modes is the set of degenerate valence band modes at the $J$-point, $V B_{A_{2}^{\prime}}^{J}$.

The simplest type of acceptor defect region consists of an enlargement of a single hole in the photonic lattice. This type of defect maintains the point group symmetry of the lattice, $C_{6 v}$. The characters of the representation of $V B_{A_{2}^{\prime}}^{J}$ under the $C_{6 v}$ point symmetry group, which we label $S_{a 1}$, are given in Table 1. $S_{a 1}$ decomposes into irreducible blocks $B_{2}^{\prime \prime} \oplus A_{2}^{\prime \prime}$. Using the projection operators, the basis functions of $V B_{A_{2}^{\prime}}^{J}$ are coupled together to form the following localized acceptor modes:

$$
\begin{aligned}
& \mathbf{B}_{A_{2}^{\prime \prime}}^{a 1}=\hat{z}\left(\cos \left(\mathbf{k}_{J_{1}} \cdot \mathbf{r}_{\perp}\right)+\cos \left(\mathbf{k}_{J_{3}} \cdot \mathbf{r}_{\perp}\right)+\cos \left(\mathbf{k}_{J_{5}} \cdot \mathbf{r}_{\perp}\right)\right), \\
& \mathbf{B}_{B_{2}^{\prime \prime}}^{a 1}=\hat{z}\left(\sin \left(\mathbf{k}_{J_{1}} \cdot \mathbf{r}_{\perp}\right)+\sin \left(\mathbf{k}_{J_{3}} \cdot \mathbf{r}_{\perp}\right)+\sin \left(\mathbf{k}_{J_{5}} \cdot \mathbf{r}_{\perp}\right)\right) .
\end{aligned}
$$

In the $X$ and $Y$-split cavities with $C_{2 v}$ symmetry, $\mathbf{B}_{A_{2}^{\prime \prime}}^{a 1}$ and $\mathbf{B}_{B_{2}^{\prime \prime}}^{a 1}$ transform as $A_{2}$ and $B_{2}$ IRREPs, respectively:

$$
\begin{aligned}
C_{6 v} & \rightarrow C_{2 v} \\
\mathbf{B}_{A_{2}^{\prime \prime}}^{a 1} & \rightarrow \mathbf{B}_{A_{2}}^{a 1}, \\
\mathbf{B}_{B_{2}^{\prime \prime}}^{a 1} & \rightarrow \mathbf{B}_{B_{2}}^{a 1} .
\end{aligned}
$$

For defect regions which strongly perturb the photonic lattice it is possible that a larger number of localized defect modes will form than can be described by the limited symmetry basis used above. This is the case for the $Y$-split cavity described below, where the defect region is composed of two enlarged holes and has a relatively deep potential well for acceptor modes. As a result, in the FDTD simulations and the PL measurements of the $Y$-split cavity an additional shallow acceptor type mode $\left(Y-A 2_{0}\right)$, not covered by the $V B_{A_{2}^{\prime}}^{J}$ symmetry basis, is present.

In order to capture more fully the possible defect modes in a deep potential well, we adopt a method based upon the observation that, for defect regions which provide a deep potential well, it is also possible that defect modes will form which are composed of unperturbed photonic crystal modes located not just at the edge of 
the band-gap, but also at other nearby (in frequency) high-symmetry $k$-points within the IBZ. In order to represent these additional localized resonant modes the unperturbed photonic crystal modes at the additional high-symmetry $k$-points must be included in the symmetry basis. For the hexagonal lattice the valence band at the $X$-point is close in frequency to the band-gap edge at the $J$-point (see Figure $1(\mathrm{~b})$ ). The symmetry basis for the $X$-point valence band-edge is the triply degenerate $V B_{A_{2}}^{X}$ basis set. The representation of $V B_{A_{2}}^{X}$ under $C_{6 v}$, labelled $S_{a 2}$, has the character values shown in Table 1 and decomposes into irreducible spaces $E_{2}$ and $A_{2}^{\prime \prime}$. The acceptor type modes formed from the $X$-point valence band modes in a symmetric defect cavity are:

$$
\begin{aligned}
& \mathbf{B}_{A_{2}^{\prime \prime}}^{a 2}=\hat{z}\left(\cos \left(\mathbf{k}_{X_{1}} \cdot \mathbf{r}_{\perp}\right)+\cos \left(\mathbf{k}_{X_{2}} \cdot \mathbf{r}_{\perp}\right)+\cos \left(\mathbf{k}_{X_{3}} \cdot \mathbf{r}_{\perp}\right)\right) \\
& \mathbf{B}_{E_{2}, 1}^{a 2}=\hat{z}\left(2 \cos \left(\mathbf{k}_{X_{1}} \cdot \mathbf{r}_{\perp}\right)-\cos \left(\mathbf{k}_{X_{2}} \cdot \mathbf{r}_{\perp}\right)-\cos \left(\mathbf{k}_{X_{3}} \cdot \mathbf{r}_{\perp}\right)\right) \\
& \mathbf{B}_{E_{2}, 2}^{a 2}=\hat{z}\left(\cos \left(\mathbf{k}_{X_{2}} \cdot \mathbf{r}_{\perp}\right)-\cos \left(\mathbf{k}_{X_{3}} \cdot \mathbf{r}_{\perp}\right)\right)
\end{aligned}
$$

The $Y$-split cavity does not have $C_{6 v}$ symmetry, but rather $C_{2 v}$ symmetry. This reduction of symmetry causes the $E_{2}$ IRREP space to split into $A_{1} \oplus A_{2}$, and the $A_{2}^{\prime \prime}$ space to transfer over into an $A_{2}$ IRREP space:

$$
\begin{aligned}
C_{6 v} & \rightarrow C_{2 v} \\
\mathbf{B}_{A_{2}^{\prime \prime}}^{a 2} & \rightarrow \mathbf{B}_{A_{2}}^{a 2,1}, \\
\mathbf{B}_{E_{2}, 1}^{a 2} & \rightarrow \mathbf{B}_{A_{2}}^{a 2,2}, \\
\mathbf{B}_{E_{2}, 2}^{a 2} & \rightarrow \mathbf{B}_{A_{1}}^{a 2} .
\end{aligned}
$$

The shallow acceptor mode $\left(Y-A 2_{0}\right)$ found in the $Y$-split FDTD simulations of the next section transforms as the $A_{2}$ IRREP under $C_{2 v}$ symmetry operations. The dominant Fourier component within the FDTD generated field pattern of $Y-A 2_{0}$ is $\mathbf{k}_{X_{1}}$, from which we can conclude that this mode is given by $\mathbf{B}_{A_{2}}^{a 2,2}$ as opposed to $\mathbf{B}_{A_{2}}^{a 2,1}$.

\section{FDTD SIMULATIONS AND PHOTOLUMINESCENCE MEASUREMENTS}

Characterization of the PC defect cavities was performed by optically pumping the MQW region within the slab waveguide and analyzing the resulting emission. The optical pumping and PL collection apparatus is described in detail in references ${ }^{18,22}$; a schematic of the measurement setup is depicted in Figure 3 . The strong polarization signature (even sub-threshold) of the deep donor (DD) $(x, y)$-dipole modes, is used as a reference point from which to classify other resonant modes. Comparing the PL measurements with FDTD simulations, the resonant peaks within the PL spectra from the defect cavities can then be given an appropriate symmetry label corresponding to the IRREP of $C_{2 v}$ under which it transforms. Resonant modes with similar transformation properties are indexed according to frequency.

Three types of defect cavities are studied (Figure 4): a symmetric $(S)$ cavity with the standard central hole removed, an $X$-split cavity in which air holes on top and bottom of the central missing hole are moved inwards a distance $\Delta y$, and a $Y$-split cavity in which the missing central hole is accompanied by two enlarged holes on either side. Actual fabricated structures, where the defect regions are surrounded by eight periods of the hexagonal lattice, contain an unintentionally introduced (radially symmetric) grading in the etched air hole size. The holes are 10-15\% larger near the center of the lattice than at the perimeter, resulting in weakly localized shallow acceptor (SA) states (described below).

Spectra from several $S$ defect cavities with lattice spacing ranging from $570 \mathrm{~nm}$ to $480 \mathrm{~nm}(r / a=0.325$, $d / a=0.3721-0.4416)$ are shown in Figure $5(\mathrm{a})$. The emission is strongly frustrated except for three peaks. The broad central set of peaks correspond to spontaneous emission from the degenerate DD dipole-like modes $\left(S-B 2_{1}, S-B 1_{0}\right)$. The guided mode band-gap extends roughly over a normalized frequency of 0.30 to 0.42 , from 


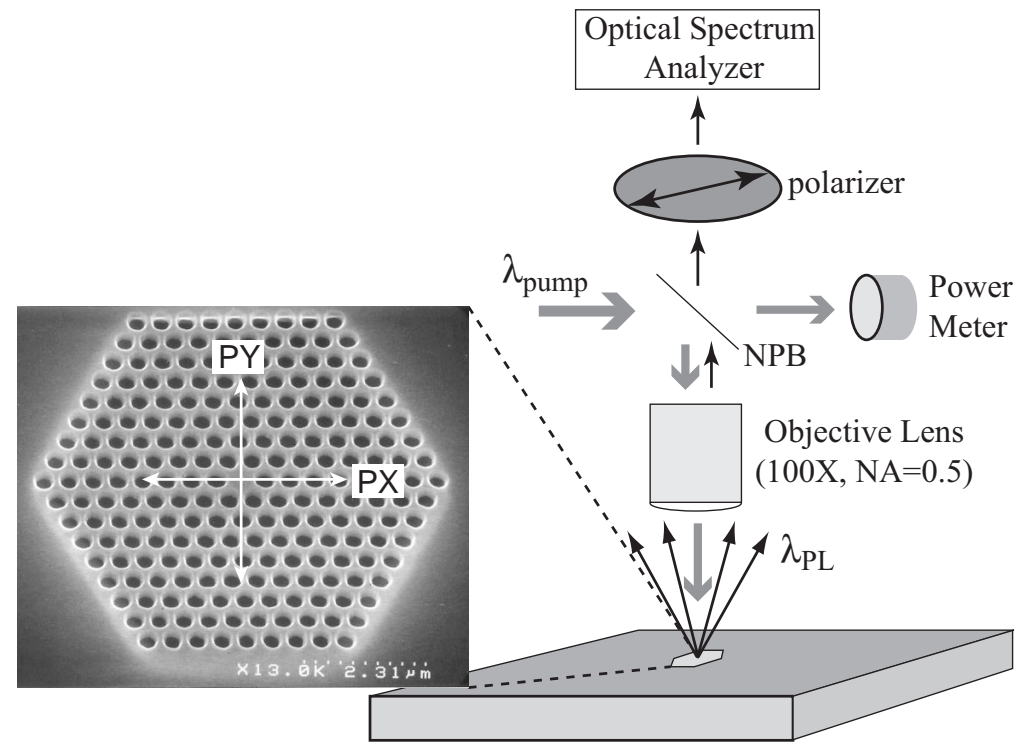

Figure 3. Schematic of the polarization selective PL measurement set-up. Inset shows a projection of the $P X$ and $P Y$ polarizer orientations onto the SEM image of a fabricated defect cavity as it would be mounted in the PL set-up.

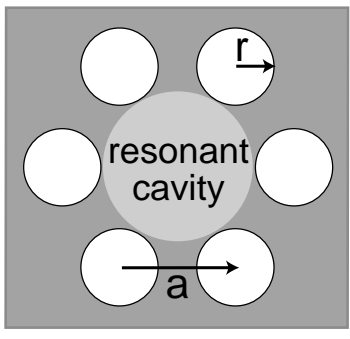

(a) Symmetric.

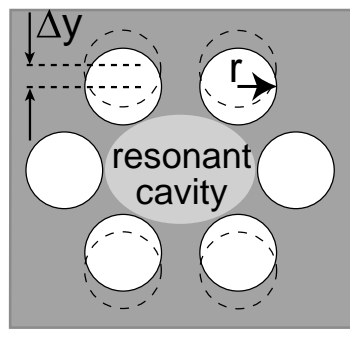

(b) $X$-split

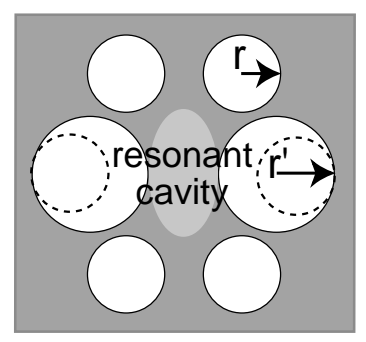

(c) $Y$-split.

Figure 4: PC cavity geometries of different symmetry.

the narrow low frequency SA mode to the high frequency shallow donor (SD) or CB mode. The slight shift in normalized frequency of the DD dipole modes versus lattice spacing is a result of the difference in normalized slab thickness, $d / a$, for the three devices. ${ }^{23}$

PL from a set of matched $S, X$-split, and $Y$-split cavities with $a=515 \mathrm{~nm}, r / a=0.35$, and $d / a=0.409$ is given in Figures 5(b)-(d). In the $X$-split cavity $\Delta y \sim 0.05 a \sim 25 \mathrm{~nm}$, and in the $Y$-split cavity $r^{\prime} / a \sim 0.51$. FDTD simulations of the frequency response of the different cavities ${ }^{14}$ are plotted as dashed lines along with the measured PL spectra. In the PL spectra of the $S$ cavity shown in Figure 5(b), the two resonance peaks are spectrally aligned with the FDTD calculated SA and DD resonances. The symmetry and dominant Fourier components of the FDTD generated degenerate DD mode field patterns shown in Figure 6(a)-(b) match those of the $x$-dipole $\left(\mathbf{B}_{E_{1}, 1}^{d 1}\right)$ and $y$-dipole $\left(\mathbf{B}_{E_{1}, 2}^{d 1}\right)$ modes from the symmetry analysis of the previous section, permitting the labels $S$ - $B 1_{0}$ ( $x$-dipole) and $S$ - $B 2_{1}$ (y-dipole) for the DD PL peak. Even the subtle difference in the inplane radiation pattern of the $(x, y)$-dipole modes as calculated numerically using FDTD is contained within 
the symmetry analysis as can be seen by the lack of a third standing wave component in the $y$-dipole $\left(\mathbf{B}_{E_{1}, 2}^{d 1}\right)$ mode. A similar correspondence relates the $\mathbf{B}_{A_{2}^{\prime \prime}}^{a 1}\left(S-A 2_{0}\right)$ and $\mathbf{B}_{B_{2}^{\prime \prime}}^{a 1}\left(S-B 2_{0}\right)$ approximate field patterns to the (accidentally) degenerate SA PL peak.

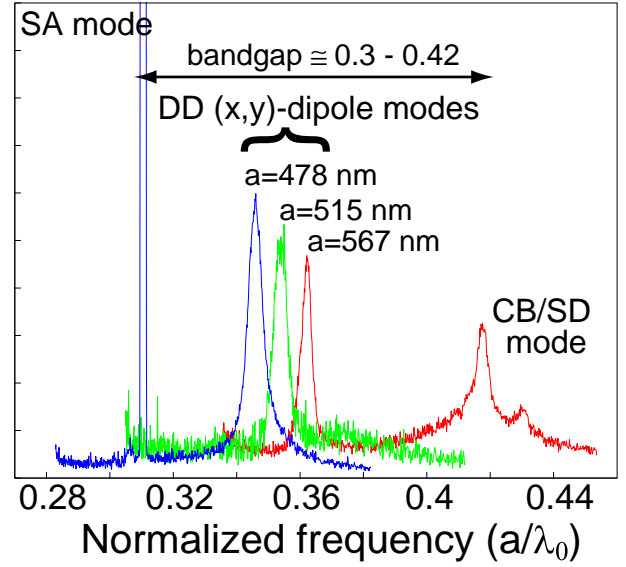

(a) Varying lattice spacing.

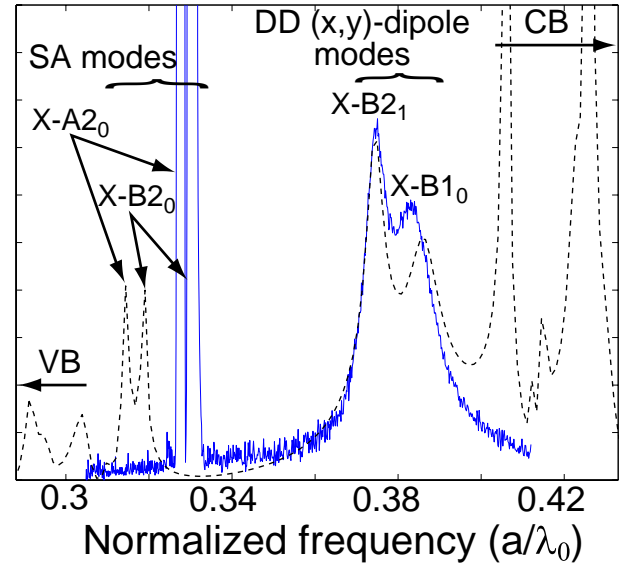

(c) $X$-split cavity.

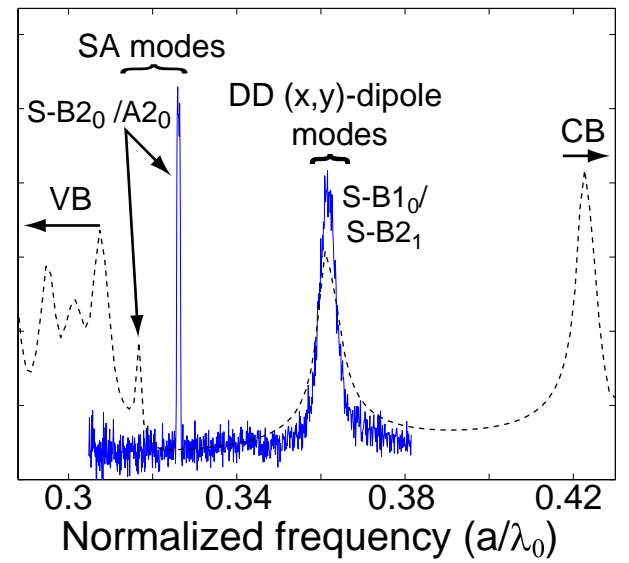

(b) $S$ cavity.

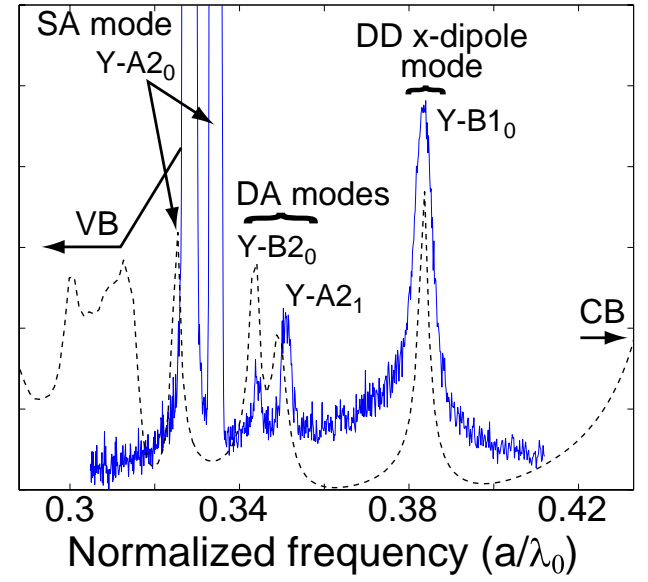

(d) $Y$-split cavity.

Figure 5. PL spectra from $S, X$-split, and $Y$-split defect cavities. In (a) the lattice spacing is varied from $a=570 \mathrm{~nm}$ to $480 \mathrm{~nm}$ with $r / a \approx 0.325$. In (b), (c), and (d): $a=515 \mathrm{~nm}, r / a=0.35$ nominally, and $d / a=0.409$. FDTD simulations are shown as dashed lines.

In the $X$-split cavity PL spectra of Figure 5(c), the DD $(x, y)$-dipole modes near $a / \lambda_{o}=0.38$ are seen to split into two distinct peaks, as predicted by symmetry arguments. Additionally, the SA peak of $S-A 2_{0}$ and $S-B 2_{0}$ in Figure 5(b) is clearly seen to separate into $X-A 2_{0}$ and $X-B 2_{0}$ peaks, respectively.

The PL spectra of the $Y$-split cavity exhibits four discrete mode peaks. The DD dipole-like modes are split, and the remaining single peak at $a / \lambda_{o}=0.36$ corresponds to the $x$-dipole mode (as measured by polarization). The two SA modes of the $S$ and $X$-split cavity are now pushed further into the the band-gap, forming deep acceptor (DA) modes $\left(Y-A 2_{1}\right.$ and $Y$-B2 $\left.2_{0}\right)$. Another SA mode, $Y-A 2_{0}$, is also present, along with a slew of modes near the valence band-edge. 


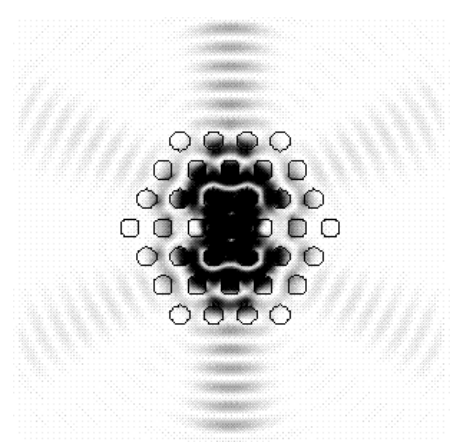

(a) In-plane $x$-dipole mode radiation pattern.

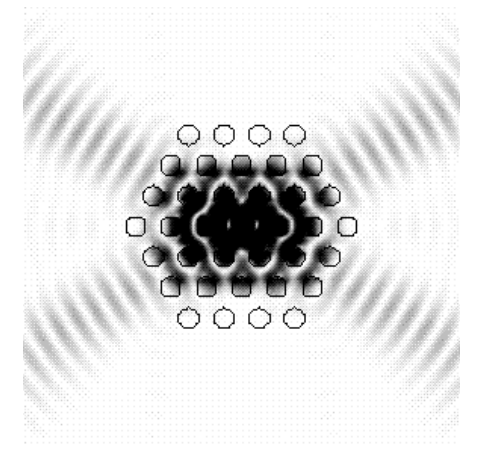

(b) In-plane $y$-dipole mode radiation pattern.

Figure 6. In-plane radiation losses (electric field amplitude saturated) of the $x$ and $y$ dipole mode (degenerate case) are shown in (a) and (b), respectively.

\section{SUMMARY}

The optical properties of defect cavities in 2D PC slab waveguides have been examined analytically through group theoretical methods, numerically through FDTD calculations, and experimentally through PL spectroscopy of fabricated devices. Using approximate fields for the dominant Fourier components of a mode, we put forth a simple process by which PC nanocavities can be designed through symmetry arguments. When combined with FDTD simulations, the two methods result in a highly descriptive model for the behavior of the defect modes of PC cavities of varying symmetry. Experimental characterization of fabricated structures largely confirms the predictions of this model, and this close correspondence illustrates the degree to which the emission properties of the resonant modes of these systems can be specified by a careful design that utilizes the techniques described in this paper.

The authors would like to thank In Kim and Denis Tishinin for the growth of the InGaAsP material used to create the PC cavities. O. Painter would like to thank Kerry Vahala for many fruitful discussions regarding group theory. K. Srinivasan thanks the Hertz Foundation for its financial support.

\section{REFERENCES}

1. D. M. Atkin, P. S. J. Russell, T. A. Birks, and P. J. Roberts, "Photonic band structure of guided Bloch modes in high index films fully etched through with periodic microstructure," J. Mod. Opt. 43, pp. 10351053, May 1996.

2. P. S. J. Russell, D. M. Atkin, and T. A. Birks, Bound Modes Of Two-Dimensional Photonic Crystal Waveguides, pp. 203-218. Kluwer Academic Publishers, The Netherlands, 1996.

3. S. G. Johnson, S. Fan, P. R. Villeneuve, J. D. Joannopoulos, and L. A. Kolodziejaki, "Guided modes in photonic crystal slabs," Phys. Rev. B 60, pp. 5751-5758, Aug. 1999.

4. A. Mekis, J. C. Chen, I. Kurland, S. Fan, P. R. Villeneuve, and J. D. Joannopoulos, "High Transmission through Sharp Bends in Photonic Crystal Waveguides," Phys. Rev. Lett. 77, pp. 3787-3790, Oct. 1996.

5. E. Chow, S. Y. Lin, J. R. Wendt, S. G. Johnson, and J. D. Joannopoulos, "Quantitative analysis of bending efficiency in photonic-crystal waveguide bends at $\lambda=1.55 \mu$ m wavelengths," Opt. Lett. 26, pp. 286-288, Mar. 2001.

6. T. Baba, N. Fukaya, and J. Yonekura, "Observation of light propagation in photonic crystal optical waveguides with bends," IEE Elec. Lett. 35, pp. 654-655, Apr. 1999.

7. M. Lončar, T. Doll, J. Vučković, and A. Scherer, "Design and Fabrication of Silicon Photonic Crystal Optical Waveguides," J. Lightwave Tech. 18, pp. 1402-1411, Oct. 2000. 
8. A. Yariv, Y. Xu, R. K. Lee, and A. Scherer, "Coupled-resonator optical waveguide: a proposal and analysis," Opt. Lett. 24, pp. 711-713, June 1999.

9. S. Olivier, C. Smith, M. Rattier, H. Benisty, C. Weisbuch, T. Krauss, R. Houdré, and U. Oesterlé, "Miniband transmission in a photonic crystal coupled-resonator optical waveguide," Opt. Lett. 26, pp. 1019-1021, July 2001.

10. C. Smith, R. De la Rue, M. Rattier, S. Olivier, H. Benisty, C. Weisbuch, T. Krauss, U. Oesterlé, and R. Houdré, "Coupled guide and cavity in a two-dimensional photonic crystal," Appl. Phys. Lett. 78, pp. 1487-1489, mar 2001.

11. S. Fan, P. R. Villeneuve, and J. D. Joannopoulos, "Channel drop filters in photonic crystals," Optics Express 3, pp. 4-11, July 1998.

12. S. Noda, A. Chutinan, and M. Imada, "Trapping and emission of photons by a single defect in a photonic bandgap structure," Nature 407, pp. 608-610, Oct. 2000.

13. J. S. Foresi, P. R. Villeneuve, J. Ferrera, E. R. Thoen, G. Steinmeyer, S. Fan, J. D. Joannopoulos, L. C. Kimerling, H. I. Smith, and E. P. Ippen, "Photonic-Bandgap microcavities in optical waveguides," Nature 390, pp. 143-145, Nov. 1997.

14. O. Painter, J. Vučković, and A. Scherer, "Defect Modes of a Two-Dimensional Photonic Crystal in an Optically Thin Dielectric Slab," J. Opt. Soc. Am. B 16, pp. 275-285, Feb. 1999.

15. R. Coccioli, M. Boroditsky, K. W. Kim, Y. Rahmat-Samii, and E. Yablonovitch, "Smallest possible electromagnetic mode volume in a dielectric cavity," IEE Proc. - Optoelectron. 145, pp. 391-397, Dec. 1998.

16. O. Painter, R. K. Lee, A. Yariv, A. Scherer, J. D. O'Brien, P. D. Dapkus, and I. Kim, "Two-Dimensional Photonic Band-Gap Defect Mode Laser," Science 284, pp. 1819-1824, June 1999.

17. O. Painter, K. Srinivasan, J. D. O'Brien, A. Scherer, and P. D. Dapkus, "Tailoring of the resonant mode properties of optical nanocavities in two-dimensional photonic crystal slab waveguides," J. Opt. A 3, pp. S161-S170, Nov. 2001.

18. O. J. Painter, Optical Nanocavities in Two-Dimensional Photonic Crystal Planar Waveguides. PhD thesis, California Institute of Technology, 2001.

19. K. Sakoda, "Symmetry, degeneracy, and uncoupled modes in two-dimensional photonic crystals," Phys. Rev. B 52, pp. 7982-7986, Sept. 1995.

20. M. Tinkham, Group Theory and Quantum Mechanics, International Series in Pure and Applied Physics, McGaw-Hill, Inc., New York, NY, 1964.

21. J. D. Joannopoulos, R. D. Meade, and J. N. Winn, Photonic Crystals, Princeton University Press, Princeton, New Jersey, 1995.

22. O. Painter and K. Srinivasan, "Polarization Properties of Dipole-Like Defect Modes in Photonic Crystal Nanocavities." Submitted to Optics Letters, July 20, 2001.

23. O. Painter, A. Husain, A. Scherer, P. T. Lee, I. Kim, J. D. O'Brien, and P. D. Dapkus, "Lithographic Tuning of a Two-Dimensional Photonic Crystal Laser Array," IEEE Photonics Tech. Lett. 12, pp. 1126-1128, Sept. 2000 . 